\title{
Avaliação da conformidade do e-SUS AB PEC segundo a certificação da SBIS/CFM
}

\author{
Rodrigo Barbalho, Lucas Postal, Raul Sidnei Wazlawick \\ Laboratório Bridge - Centro Tecnológico - Universidade Federal de Santa Catarina \\ (UFSC) \\ Caixa Postal 476 - 88010-970 - Florianópolis, SC - Brasil \\ \{rodrigo.barbalho, postal, raul\} @bridge.ufsc.br
}

\begin{abstract}
In the health information registry, the Electronic Health Record System (EHRs) and the Electronic Patient Record (EPR) have been gaining ground. Maintaining data security and confidentiality is a challenge. Then, SBIS joined forces with CFM and created the certification model for EHRs. This paper evaluates the e-SUS AB PEC system provided by the Ministry of Health (MH) regarding its compliance with the requirements of Structure, Content and Functionality and Security Assurance Level 1 (SAL1). For this purpose, a scale for conformity classification has been defined. Then the tests provided in the manual of tests and analyzes were applied, where it was found that only $32.5 \%$ of the requirements were classified as fully implemented.
\end{abstract}

Resumo. No registro de informações de saúde, o Sistema de Registro Eletrônico de Saúde (S-RES) e Prontuário Eletrônico do Paciente (PEP) vêm ganhando espaço. Manter a segurança e a confidencialidade dos dados é um desafio. Então, a SBIS ${ }^{l}$ uniu forças com o $C_{F}^{2}$ e criaram o modelo de certificação para S-RES. Este trabalho avalia o sistema e-SUS AB PEC fornecido pelo Ministério da Saúde (MS) quanto a sua conformidade em relação aos requisitos de Estrutura, Conteúdo e Funcionalidades e Nivel de Garantia de Segurança 1 (NGS1). Para isso, foi definida uma escala para classificação da conformidade. Em seguida foram aplicados os testes disponibilizados no manual de ensaios e análises onde foi constatado que apenas 32,5\% dos requisitos foram classificados como totalmente implementado.

\section{Introdução}

Registro Eletrônico em Saúde (RES) é um repositório de informação a respeito da saúde de indivíduos, numa forma processável eletronicamente. Sistema de Registro Eletrônico de Saúde (S-RES) é um sistema para registro, recuperação e manipulação das informações de um RES [ABNT, 2008][ABNT, 2013]. Um Prontuário Eletrônico é um exemplo de S-RES e contém informações sobre a saúde passada, presente e futura e as condições físicas e mentais de um indivíduo. Essas informações residem em um computador no qual é utilizado para captar, transmitir, receber, armazenar, distribuir, interligar e manipular dados para os principais objetivos de um serviço de atenção à saúde [Murphy, Hanken e Waters, 1999].

\footnotetext{
${ }^{1}$ Sociedade Brasileira de Informática em Saúde

${ }^{2}$ Conselho Federal de Medicina

${ }^{3}$ Sistema de Prontuário Eletrônico do Cidadão para Atenção Básica
} 
A principal função de um prontuário eletrônico é apoiar os usuários através da acessibilidade aos dados completos e exatos, de alertas, lembretes, notificações, sistemas de apoio à decisão clínica e dos vínculos ao conhecimento médico.

O Ministério da Saúde (MS), com o objetivo de informatizar os sistemas de saúde no território nacional, criou a Política Nacional de Informação e Informática em Saúde (PNIIS)[Ministério da Saúde, 2016] e como parte dela estabeleceu a estratégia eSUS Atenção Básica (AB). A estratégia é composta de um conjunto de ações que tem como objetivo melhorar a qualidade da informação em saúde através de uma série de políticas.

Dentro da estratégia e-SUS AB, surge o Prontuário Eletrônico do Cidadão (PEC), como um dos pilares dessa política. Esse sistema é desenvolvido e distribuído gratuitamente para todos os municípios brasileiros para informatizar as Unidades Básicas de Saúde (UBS). No contexto de sistemas de informação de saúde, critérios como segurança, confiabilidade, confidencialidade e legalidade do registro eletrônico de saúde, tornam-se fundamentais.

Com interesse na qualidade dos S-RES e também visando garantir a qualidade desses sistemas e das informações geradas através deles, associações médicas de vários países trabalham em modelos para realizarem avaliações da qualidade de sistemas de saúde, como é o caso do Electronic Medical Record Adoption Model (EMRAM) ${ }^{4}$, desenvolvido pela Health Information and Management Systems Society (HIMSS), nos Estados Unidos. Este modelo classifica os sistemas em 7 níveis de acordo com a sua capacidade de implementar funções de registros eletrônicos de saúde. O objetivo é auxiliar hospitais que queiram adotar sistemas de informação ou que pretendem melhorar a sua gestão com base neste modelo reconhecido mundialmente.

No Brasil, a Sociedade Brasileira de Informática em Saúde (SBIS), em conjunto com o Conselho Federal de Medicina (CFM), vêm trabalhando ao longo dos anos em normas e padrões para melhorar a qualidade das informações em sistemas de informação em saúde. A resolução $n^{0}$ 1821/2007, citou pela primeira vez um Manual de Certificação para S-RES. Era o início de uma política focada em melhorar a qualidade de sistemas de informação em saúde no território nacional. Mais tarde, isso gerou todo o processo de certificação de sistemas de saúde, realizado pela SBIS.

Além de permitir a avaliação de S-RES existentes, o modelo de certificação da SBIS/CFM também pode ser utilizado para nortear o desenvolvimento de novos S-RES. Em Garcia et al. (2010), foi elaborada a modelagem de um S-RES considerando os requisitos obrigatórios para obtenção da certificação. Em Virginio et al. (2014), foi realizada a avaliação do S-RES de um hospital universitário, em uma cidade no interior de São Paulo, também de acordo com os requisitos da certificação SBIS/CFM.

Em César et al. (2016) foi realizada a avaliação do e-SUS AB, versão de treinamento disponibilizada pelo Ministério da Saúde. Foram considerados os requisitos de Estrutura, Conteúdo e Funcionalidades e Nível de Garantia de Segurança 1 da certificação SBIS/CFM versão 3.3. No entanto, os autores não evidenciaram a versão do

\footnotetext{
${ }^{4}$ http://www.himssanalytics.org/emram
} 
PEC que foi utilizada para testes, se utilizaram o e-SUS AB PEC ou CDS, ou se foram avaliados todos os requisitos obrigatórios e recomendados.

O objetivo desta pesquisa foi de avaliar a conformidade do e-SUS AB PEC versão 2.1.08 de 2016 fornecido pelo Ministério da Saúde com o modelo de certificação da SBIS/CFM através da implementação dos cenários de teste descritos no Manual Operacional de Ensaios e Análises [SBIS, 2016b], para verificar a aderência do sistema aos requisitos considerados obrigatórios para a certificação.

\section{Materiais e Métodos}

A avaliação do e-SUS AB foi realizada em etapas. Na primeira etapa foi definido o método de avaliação. A definição e configuração do ambiente de teste foi realizada na segunda etapa. Já na terceira e última etapa foram realizados os testes.

\subsection{Definição do método de avaliação}

Baseado no Manual de Certificação para S-RES versão 4.2 (Edição 2016) e no Manual Operacional de Ensaios e Análises para Certificação de S-RES versão 2.1 de 2016, ambos disponibilizados para download no site da SBIS, foi constatado que o PEC se enquadra na categoria assistencial, a qual é voltada à assistência à saúde de indivíduos.

O método de avaliação é similar ao utilizado por auditores da SBIS durante o processo de certificação. Foram considerados os requisitos de Estrutura, Conteúdo e Funcionalidades e Nível de Garantia de Segurança 1 (NGS1) da certificação SBIS/CFM versão 4.2. Não foram avaliados os requisitos referentes à Gestão Eletrônica de Documentos (GED) e nem os requisitos pertencentes ao Nível de Garantia de Segurança 2 (NGS2).

Para cada requisito obrigatório para obtenção da certificação, foram realizados os testes descritos no Manual Operacional de Ensaios e Análises. No manual de certificação, os requisitos são classificados como obrigatórios, opcionais ou que não se aplicam. No contexto desta pesquisa, apenas os requisitos definidos como obrigatórios foram avaliados.

Alguns scripts de testes são fracionados em cenários distintos tornando possível que o S-RES alcance um resultado esperado e outro não. Sendo assim, definimos uma escala para avaliação dos requisitos:

- NA - Não se aplica: quando não se aplicar ao contexto/arquitetura do e-SUS AB PEC;

- $\quad \mathrm{N}$ - Não implementado: quando o e-SUS AB PEC não for capaz de atender nenhum dos resultados esperados;

- P - Parcialmente implementado: quando o e-SUS AB PEC for capaz de atender pelo menos um dos resultados esperados;

- $\mathrm{T}$ - Totalmente implementado: quando e-SUS AB PEC for capaz de atender o requisito em sua totalidade. 


\subsection{Configuração do ambiente de testes}

Para a realização dos testes de conformidade foi utilizado o sistema e-SUS AB PEC Treinamento, versão 2.1.08, disponibilizado para download no site do Ministério da Saúde. Nesta versão é instalado o banco de dados PostgreSQL 9.3 com o pgAdmin III.

Após a instalação, foi importado um arquivo no formato .xml com dados fictícios para que o sistema pudesse ser configurado e preparado para realização dos testes. A configuração do servidor de testes utilizado é superior à configuração mínima recomendada pelo DAB. A Tabela 1 apresenta as diferenças de configuração.

Tabela 1. Diferença de configurações

\begin{tabular}{|l|l|l|}
\hline & Configuração recomendada (DAB) & Configuração utilizada \\
\hline Processador & $2,0 \mathrm{GHz}$ dual-core & $2,7 \mathrm{GHz}$ dual-core \\
\hline Memória RAM & $4,0 \mathrm{~GB}$ & $8,0 \mathrm{~GB}$ \\
\hline Espaço em disco & $50 \mathrm{~GB}$ & $+100 \mathrm{~GB}$ \\
\hline Sistema operacional & $32 \mathrm{bits}$ & 64 bits \\
\hline
\end{tabular}

\subsection{Realização dos testes}

Os testes foram executados em sua maioria utilizando um computador desktop com as configurações descritas acima. Para os testes de alguns requisitos que tratavam sobre documentos do S-RES, foi utilizado como referência o site do Departamento da Atenção Básica (DAB) e os manuais disponibilizados através dele.

\section{Resultados das avaliações}

Esta seção apresenta os resultados obtidos com a avaliação dos requisitos definidos como obrigatórios para obtenção da certificação da SBIS. Para cada um destes requisitos, o manual operacional de ensaios e análises dispõe de um cenário de teste. Ao ser executado deverá revelar se o S-RES atende ao requisito ou não. Há uma ressalva para os requisitos classificados como "não se aplica".

\subsection{Avaliação referente ao nível de garantia de segurança 1 (NGS1)}

No NGS1 - Assistencial, o S-RES deve contemplar um total de 59 requisitos. A Tabela 2 apresenta os requisitos obrigatórios e o resultado da avaliação. 
Tabela 2. Requisitos obrigatórios para NGS1 e sua classificação

\begin{tabular}{|l|c|l|l|l|l|c|}
\hline Requisito & $\begin{array}{l}\text { Resultado } \\
\text { da avaliação }\end{array}$ & Requisito & $\begin{array}{l}\text { Resultado da } \\
\text { avaliação }\end{array}$ & Requisito & $\begin{array}{l}\text { Resultado da } \\
\text { avaliação }\end{array}$ \\
\hline NGS1.01.01 & P & NGS1.05.02 & N & NGS1.09.01 & P \\
\hline NGS1.02.01 & T & NGS1.05.03 & N & NGS1.09.02 & N \\
\hline NGS1.02.02 & T & NGS1.06.01 & N & NGS1.09.04 & N \\
\hline NGS1.02.03 & T & NGS1.06.02 & N & NGS1.09.05 & N \\
\hline NGS1.02.04 & P & NGS1.06.03 & T & NGS1.09.07 & NA \\
\hline NGS1.02.05 & N & NGS1.06.04 & N & NGS1.09.08 & N \\
\hline NGS1.02.06 & T & NGS1.06.05 & N & NGS1.09.09 & N \\
\hline NGS1.02.08 & N & NGS1.06.06 & N & NGS1.09.10 & NA \\
\hline NGS1.03.01 & T & NGS1.06.07 & T & NGS1.09.11 & NA \\
\hline NGS1.03.02 & P & NGS1.06.08 & T & NGS1.09.12 & T \\
\hline NGS1.04.01 & P & NGS1.07.01 & T & NGS1.09.13 & N \\
\hline NGS1.04.02 & T & NGS1.07.05 & T & NGS1.09.14 & N \\
\hline NGS1.04.03 & T & NGS1.07.06 & T & NGS1.10.1 & N \\
\hline NGS1.04.04 & P & NGS1.07.10 & T & NGS1.10.3 & P \\
\hline NGS1.04.05 & T & NGS1.07.11 & NA & NGS1.12.01 & T \\
\hline NGS1.04.06 & P & NGS1.08.01 & T & NGS1.12.07 & N \\
\hline NGS1.04.07 & N & NGS1.08.02 & P & NGS1.13.03 & N \\
\hline NGS1.04.08 & N & NGS1.08.04 & N & NGS1.13.04 & N \\
\hline NGS1.04.11 & N & NGS1.08.05 & N & NGS1.13.06 & N \\
\hline NGS1.05.01 & P & NGS1.08.06 & N & $--/--~$ & $--/--~$ & \\
\hline
\end{tabular}

Alguns requisitos foram classificados como "Não se aplica" uma vez que possuem condições para a sua existência que estão fora de contexto/arquitetura do sistema avaliado. A seguir são apresentados estes requisitos, suas condições e a justificativa para a classificação:

- NGS1.07.11 - Condição: "S-RES ofertado na modalidade "S-RESaaS" (S-RES as a Service).”. Justificativa: o e-SUS AB PEC não funciona como um serviço;

- NGS1.09.07 e NGS1.09.10 - Condição: "S-RES composto por componentes distribuídos.”. Justificativa: o e-SUS AB PEC não possui uma arquitetura para funcionar de forma distribuída; 
- NGS1.09.11 - Condição: "Possibilidade de importação automática de dados de dispositivos externos informação de saúde.". Justificativa: o e-SUS AB PEC não faz a importação de dados de dispositivos externos.

Conforme apresentado na Figura 1, dos 59 requisitos definidos como obrigatórios no manual de certificação da SBIS, o e-SUS AB PEC implementa totalmente 18 deles $(30,5 \%)$. Parcialmente implementados foram registrados 10 requisitos (16,9\%). Não implementados somaram $27(45,8 \%)$ e 3 requisitos $(6,8 \%)$ foram classificados como não se aplica.

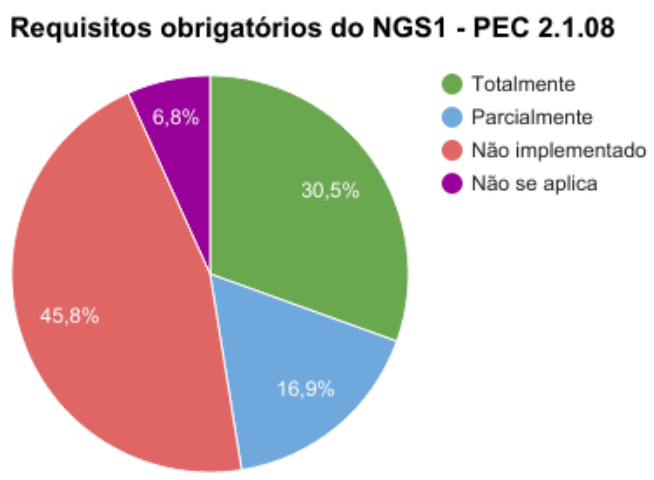

Figura 1. Quantitativo de requisitos e sua classificação

\subsection{Avaliação dos requisitos de estrutura e conteúdo (ESTR)}

Nesta seção são apresentados, conforme a Tabela 3, os resultados obtidos com a avaliação dos 29 requisitos obrigatórios do modelo de estrutura e conteúdo.

Tabela 3. Requisitos obrigatórios de estrutura e conteúdo, e sua classificação

\begin{tabular}{|c|c|c|c|c|c|}
\hline Requisito & $\begin{array}{l}\text { Resultado da } \\
\text { avaliação }\end{array}$ & Requisito & $\begin{array}{l}\text { Resultado da } \\
\text { avaliação }\end{array}$ & Requisito & $\begin{array}{l}\text { Resultado da } \\
\text { avaliação }\end{array}$ \\
\hline ESTR.01.01 & $\mathbf{T}$ & ESTR.03.03 & $\mathbf{T}$ & ESTR.05.01 & $\mathbf{N}$ \\
\hline ESTR.01.03 & $\mathbf{T}$ & ESTR.03.05 & $\mathbf{N}$ & ESTR.05.04 & $\mathbf{P}$ \\
\hline ESTR.01.04 & $\mathbf{N}$ & ESTR.03.07 & $\mathbf{T}$ & ESTR.05.06 & $\mathbf{P}$ \\
\hline ESTR.01.06 & $\mathbf{T}$ & ESTR.03.08 & $\mathbf{T}$ & ESTR.05.09 & $\mathbf{P}$ \\
\hline ESTR.02.02 & $\mathbf{T}$ & ESTR.03.09 & $\mathbf{T}$ & ESTR.05.13 & $\mathbf{N}$ \\
\hline ESTR.02.03 & $\mathbf{N}$ & ESTR.03.10 & $\mathbf{P}$ & ESTR.05.14 & $\mathbf{P}$ \\
\hline ESTR.02.05 & $\mathbf{P}$ & ESTR.03.11 & $\mathbf{P}$ & ESTR.09.02 & $\mathbf{P}$ \\
\hline ESTR.02.06 & $\mathbf{T}$ & ESTR.04.01 & $\mathbf{T}$ & ESTR.09.03 & $\mathbf{T}$ \\
\hline ESTR.02.10 & $\mathbf{T}$ & ESTR.04.02 & $\mathbf{P}$ & ESTR.09.04 & $\mathbf{T}$ \\
\hline ESTR.02.11 & $\mathbf{T}$ & ESTR.04.06 & $\mathbf{P}$ & $--/--$ & $--/--$ \\
\hline
\end{tabular}


Neste modelo, conforme apresentado na Figura 2, o e-SUS AB PEC implementa de forma total 14 requisitos $(48,3 \%)$. Parcialmente implementados somam 10 requisitos $(34,5 \%)$. Apenas 5 requisitos $(17,2 \%)$ foram classificados como não implementados.

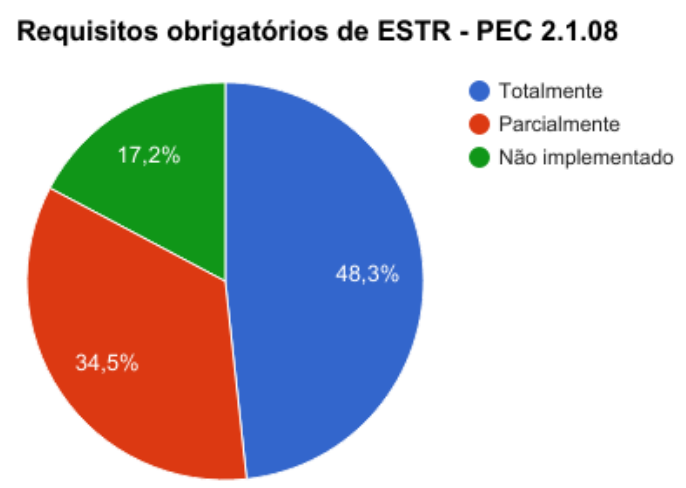

Figura 2. Quantitativo de requisitos de estrutura e conteúdo e sua classificação

\subsection{Avaliação dos requisitos de funcionalidade (FUNC)}

Totalizando também 29 requisitos obrigatórios, nos quais foram executados os testes contidos no manual de ensaios e análises, a Tabela 4 apresenta os resultados obtidos com a avaliação dos requisitos do modelo de funcionalidade.

Tabela 4. Requisitos obrigatórios de funcionalidade e sua classificação

\begin{tabular}{|l|c|l|l|l|l|l|}
\hline Requisito & $\begin{array}{l}\text { Resultado da } \\
\text { avaliação }\end{array}$ & Requisito & $\begin{array}{l}\text { Resultado da } \\
\text { avaliação }\end{array}$ & Requisito & $\begin{array}{l}\text { Resultado da } \\
\text { avaliação }\end{array}$ \\
\hline FUNC.01.04 & P & FUNC.05.01 & P & FUNC.16.02 & N \\
\hline FUNC.02.01 & T & FUNC.06.01 & T & FUNC.17.01 & P \\
\hline FUNC.02.03 & T & FUNC.07.01 & T & FUNC.18.04 & P \\
\hline FUNC.04.01 & P & FUNC.08.02 & P & FUNC.18.09 & N \\
\hline FUNC.04.03 & N & FUNC.09.01 & P & FUNC.23.01 & N \\
\hline FUNC.04.05 & N & FUNC.09.02 & P & FUNC.23.02 & N \\
\hline FUNC.04.06 & T & FUNC.09.03 & P & FUNC.25.02 & N \\
\hline FUNC.04.07 & T & FUNC.09.04 & N & FUNC.25.04 & N \\
\hline FUNC.04.08 & N & FUNC.11.02 & NA & FUNC.27.01 & NA \\
\hline FUNC.04.09 & P & FUNC.16.01 & N & & $---/--~$ & F--- \\
\hline
\end{tabular}

Alguns requisitos foram classificados como "Não se aplica" uma vez que possuem condições para a sua existência que estão fora de contexto/arquitetura do eSUS AB PEC. A seguir são apresentados estes requisitos, suas condições e a justificativa para a classificação: 
- FUNC.11.02 - Condição: "S-RES registrar áudio e/ou imagem médica ou odontológica.". Justificativa: o PEC não registra áudio e/ou imagem médica ou odontológica;

- FUNC.27.01 - Condição: "Existir uma versão anterior do mesmo S-RES já certificada pelo processo SBIS/CFM.”. Justificativa: nenhuma versão do PEC é certificada pelo modelo SBIS/CFM.

Conforme apresentado na Figura 3, dos 29 requisitos definidos como obrigatórios no manual de certificação da SBIS, o e-SUS AB PEC implementa totalmente 6 deles (20,7\%). Parcialmente implementados foram registrados 10 requisitos (34,5\%). Não implementados somam 11 (37,9\%) e 2 requisitos $(6,9 \%)$ foram classificados como não se aplica.

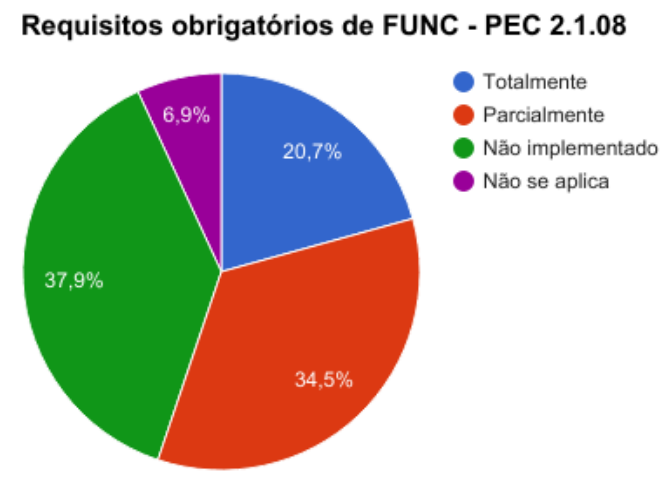

Figura 3. Quantitativo de requisitos de funcionalidade e sua classificação

\subsection{Panorama geral}

Para a certificação oficial, a classificação "Parcialmente implementado" utilizada nesta pesquisa não existe. O S-RES deve ser capaz de contemplar totalmente o requisito ou não é considerado como atendido. Na Figura 4 apresentamos uma visão geral considerando somente os requisitos classificados como "Totalmente implementados" em relação ao total de requisitos da respectiva categoria.

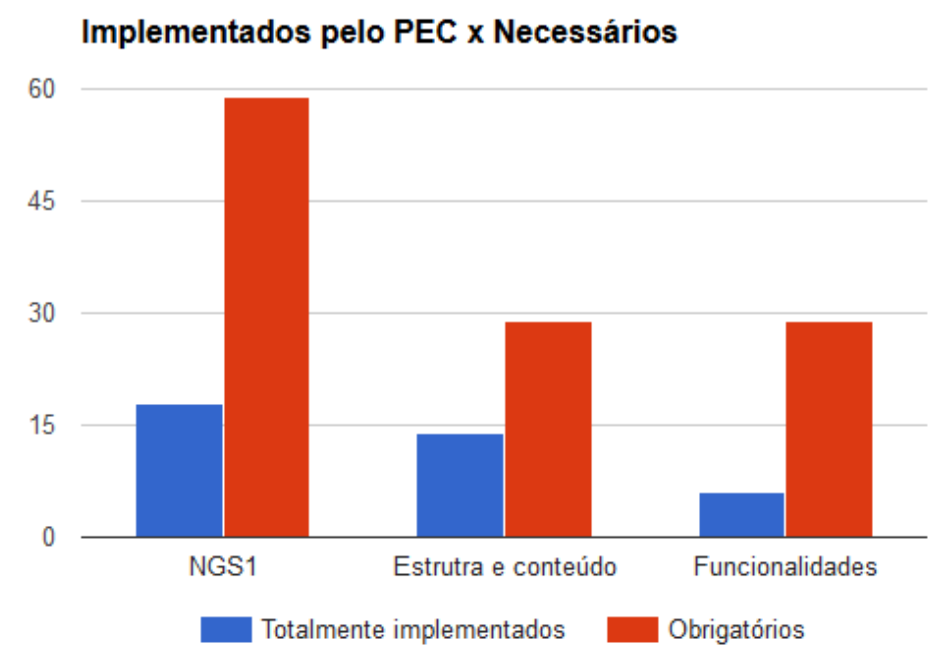

Figura 4. Requisitos implementados em relação aos necessários 


\section{Ameaças à validade}

Tendo em vista que este trabalho visa mostrar uma visão geral do nível de conformidade do e-SUS AB PEC e não realizar uma auditoria formal, não fez-se necessária a participação de um auditor autorizado pela SBIS. Com isso, é possível que o resultado possa não ter a mesma credibilidade se fosse realizada uma auditoria oficial.

Também não houve a participação de um profissional da área de saúde para confirmar se os dados clínicos foram inseridos corretamente durante a execução dos testes, o que também pode ter comprometido os resultados apresentados.

Por fim, o uso do sistema na versão de treinamento pode ser um complicador pois não apresenta todas as funcionalidades se comparada à versão de produção.

\section{Conclusão}

A preocupação em garantir a qualidade e a segurança dos S-RES é fundamental e deve ser adotada. O processo de certificação tem garantias suficientes de qualidade e segurança para qualquer S-RES. Por isso, é importante que os S-RES estejam alinhados com os requisitos exigidos para a certificação.

Sobre os resultados apresentados, segundo análise dos autores, o e-SUS AB PEC não implementa todos os requisitos obrigatórios em sua totalidade. Fato este que reprovaria o sistema caso fosse submetido ao processo de certificação da SBIS/CFM. Ainda assim, a avaliação pode ser considerada positiva, considerando-se que a equipe de desenvolvimento do e-SUS AB PEC juntamente com o Ministério da Saúde têm em mãos um material detalhado e que pode auxiliar no planejamento para que mais requisitos alcancem a classificação de totalmente implementado, aumentando a qualidade e segurança do sistema e dos seus dados.

Há também um número considerável de requisitos classificados como parcialmente implementados. Se estes requisitos atingissem a classificação esperada, ou seja, passassem de parcialmente para totalmente implementados, o sistema elevaria o índice de $38(32,5 \%)$ para $73(62,4 \%)$ requisitos totalmente implementados.

Por fim, lembramos que a certificação é válida para uma determinada versão do sistema e que a avaliação de cada nova versão tem um custo e um processo envolvido. O Ministério da Saúde costuma disponibilizar uma versão quase que mensalmente, na maioria dos casos para correção de bugs e problemas encontrados. Manter uma versão sempre certificada representaria um custo considerável. Além disso, não há interesse por parte do Ministério da Saúde em submeter o PEC ao processo de certificação, mas há uma preocupação em manter o sistema cada vez mais aderente aos requisitos da certificação.

\subsection{Trabalhos futuros}

Como trabalhos futuros, destaca-se a possibilidade de incluir os requisitos de Estrutura, Conteúdo e Funcionalidades e Nível de Garantia de Segurança 1 (NGS1) classificados como recomendados para a certificação, uma vez que estes não fizeram parte do escopo deste trabalho. Também avaliar os requisitos referentes à Gestão Eletrônica de Documentos (GED) e pertencentes ao Nível de Garantia de Segurança 2 (NGS2). Outra possibilidade é a de aplicar novamente a avaliação utilizando versões futuras do e-SUS 
AB PEC com o objetivo de avaliar a evolução da conformidade do sistema com os requisitos do Manual de Certificação para S-RES. Também, avaliar continuamente a conformidade de acordo com novas versões dos manuais.

\section{Referências}

Virginio, L., Ricarte, I., Souza J. Marques, P. (2014). Avaliação do S-RES de um hospital universitário baseada no processo de certificação SBIS/CFM, In: Congresso Brasileiro de Informática em Saúde - CBIS 2014, Santos - SP.

Garcia, D., Simões, P., Bhrem, M., Goularte, F., Spíndola de Bem, F., Rosa, M., Casagrande, M. (2010). Perspectivas da Utilização dos Requisitos Mandatórios de Conteúdo e Estrutura do Processo de Certificação de Software em Saúde Brasileiro na Modelagem de um Sistema e Registro Eletrônico em Saúde, In: Congresso Sul Brasileiro de Computação - SULCOMP 2010.

César, H., Menezes-Reis, R., Ferranti, J., Alves, D., Azevedo-Marques, P. (2016). Auditoria do e-SUS AB segundo a Certificação para S-RES SBIS/CFM, In: Congresso Brasileiro de Informática em Saúde - CBIS 2016. Goiânia - GO.

SBIS (2016a). Manual de certificação para sistemas de registro eletrônico em saúde v4.2. Disponível em: http://www.sbis.org.br/certificacao/Manual_Certificacao_SBISCFM_2016_v4-2.pdf, último acesso em: 19/03/2017, às 17:02.

SBIS (2016b). Manual operacional de ensaios e análises para certificação de S-RES versão 2.1. Disponível em: http://www.sbis.org.br/certificacao/Manual_OperEnsAnal_SBIS-CFM_2016_v21.pdf, último acesso em: 19/03/2017, às 17:02.

Murphy, G., Hanken, M. A. and Waters, K. (1999). Electronic health records: Changing the vision. Saunders WB Co.

Associação Brasileira de Normas Técnicas. ABNT ISO/TR 20.514: Informática em saúde - Registro eletrônico de saúde - Definição, escopo e contexto. Rio de Janeiro, 2008 .

Associação Brasileira de Normas Técnicas. ABNT NBR ISO 18.308: Informática em saúde - Requisitos para uma arquitetura de registro eletrônico de saúde. Rio de Janeiro, 2013.

BRASIL. Conselho Federal de Medicina. Resolução nº 1821, de 2007. Normas técnicas concernentes à digitalização e uso dos sistemas informatizados para a guarda e manuseio de documentos dos prontuários dos pacientes, autorizando a eliminação do papel e a troca de informação identificada em Saúde. Disponível em: http://www.portalmedico.org.br/resolucoes/cfm/2007/1821_2007.htm, último acesso em: 21/03/2017, às 19:02.

Ministério da Saúde. Política Nacional de Informação e Informática em Saúde. Disponível em: http://bvsms.saude.gov.br/bvs/publicacoes/politica_nacional_infor_informatica_saud e_2016.pdf, último acesso em 05/05/2017. 